\title{
Ultrafast far-infrared optics of carbon nanotubes
}

\author{
C. Frischkorn, T. Kampfrath, L. Perfetti, K. von Volkmann, and M. Wolf \\ Freie Universität Berlin, Fachbereich Physik, Arnimallee 14, 14195 Berlin, Germany
}

\begin{abstract}
The optical properties of single-wall carbon nanotube sheets in the far-infrared (FIR) spectral range from few $\mathrm{THz}$ to several tens of $\mathrm{THz}$ have been investigated with terahertz spectroscopy both with static measurements elucidating the absorption mechanism in the FIR and with time-resolved experiments yielding information on the charge carrier dynamics after optical excitation of the nanotubes. We observe an overall depletion of the dominating broad absorption peak at around $4 \mathrm{THz}$ when the nanotubes are excited by a short visible laser pulse. This finding excludes particle-plasmon resonances as absorption mechanism and instead shows that interband transitions in tubes with an energy gap of $\sim 10 \mathrm{meV}$ govern the far-infrared conductivity. A simple model based on an ensemble of two-level systems naturally explains the weak temperature dependence of the far-infrared conductivity by the tube-to-tube variation of the chemical potential. Furthermore, the time-resolved measurements do not show any evidence of a distinct free-carrier response which is attributed to the photogeneration of strongly bound excitons in the tubes with large energy gaps. The rapid decay of a featureless background with pronounced dichroism is associated with the increased absorption of spatially localized charge carriers before thermalization is completed.
\end{abstract}

Keywords: ultrafast phenomena, carbon nanotubes, graphite, THz spectroscopy, electron-phonon coupling, charge localization, interband transition, chemical potential

\section{INTRODUCTION}

Single-wall carbon nanotubes (NTs) can be pictured as rolled-up hexagonal sheets of carbon atoms. ${ }^{1}$ Depending on the wrapping direction and the diameter of the resulting entities, these tubes exhibit either semiconducting or purely metallic charge transport properties. The energy gap of semiconducting tubes with a diameter of $1 \mathrm{~nm}$ lies in the $10-\mathrm{meV}$ or 1-eV range for so-called small- and large-gap tubes, respectively. ${ }^{2}$ Carbon nanotubes at the focus of nanotechnology research ${ }^{3}$ and as prototypes of an almost one-dimensional solid have attracted significant attention in particular due to this broad range of conducting properties. Furthermore, the interaction between an individual NT and its local environment, such as other tubes or adsorbed molecules, can cause an opening of a small energy gap. ${ }^{4}$ Consequently, due to this high sensitivity of the electronic structure to environmental changes along with the large tube surface, NTs have been suggested as effective chemical sensors. ${ }^{5}$ For instance, covalently bound side chains to the NT wall ${ }^{6}$ and also the doping level of the tubes have been shown to crucially influence the absorption of FIR light. ${ }^{7-9}$ On the other hand, in nanoelectronics, it is essential to know the charge carrier dynamics of the used nanoscale elements such as carbon NTs, which promise unique applications due to their small physical diameters and the high electrical breakdown in conjunction with the various conductivities. In particular, charge localization potentially induced by defects or impurities significantly affect the electron mobility. ${ }^{10}$ Hence, the question of how charge carriers dissipate their excess energy and relax their total current after a driving electric field has been switched off is of fundamental importance for electronic applications.

Terahertz (THz) spectroscopy and more specifically the time-resolved variant of this spectroscopic method represents a very potential tool to address these issues of FIR absorption mechanism and the charge carrier dynamics. Precisely the latter near the Fermi level determine the transport properties of electrons and holes of a certain sample. In time-resolved $\mathrm{THz}$ experiments, a visible pump pulse excites the charge carriers and a subsequent $\mathrm{THz}$ pulse probes the low-energy response of the system $(2 \pi \hbar \cdot 1 \mathrm{THz}=4.1 \mathrm{meV})$. The transmitted ultrashort $\mathrm{THz}$ pulses (0.1 to $1 \mathrm{ps}$ in duration) are detected as electric field of the radiation, i.e., resolving its amplitude and phase. Consequently, full information of the complex dielectric function and, equivalently, conductivity can be obtained without the aid of Kramers-Kronig relations.

Further author information: www.physik.fu-berlin.de/ femtoweb

e-mail: christian.frischkorn@physik.fu-berlin.de, telephone: +49 3083856053

Ultrafast Phenomena in Semiconductors and Nanostructure Materials XIII,

edited by Kong-Thon Tsen, Jin-Joo Song, Markus Betz, Abdulhakem Y. Elezzabi,

Proc. of SPIE Vol. 7214, 72140A - (C) 2009 SPIE · CCC code: 0277-786X/09/\$18 · doi: 10.1117/12.809252

Proc. of SPIE Vol. 7214 72140A-1 


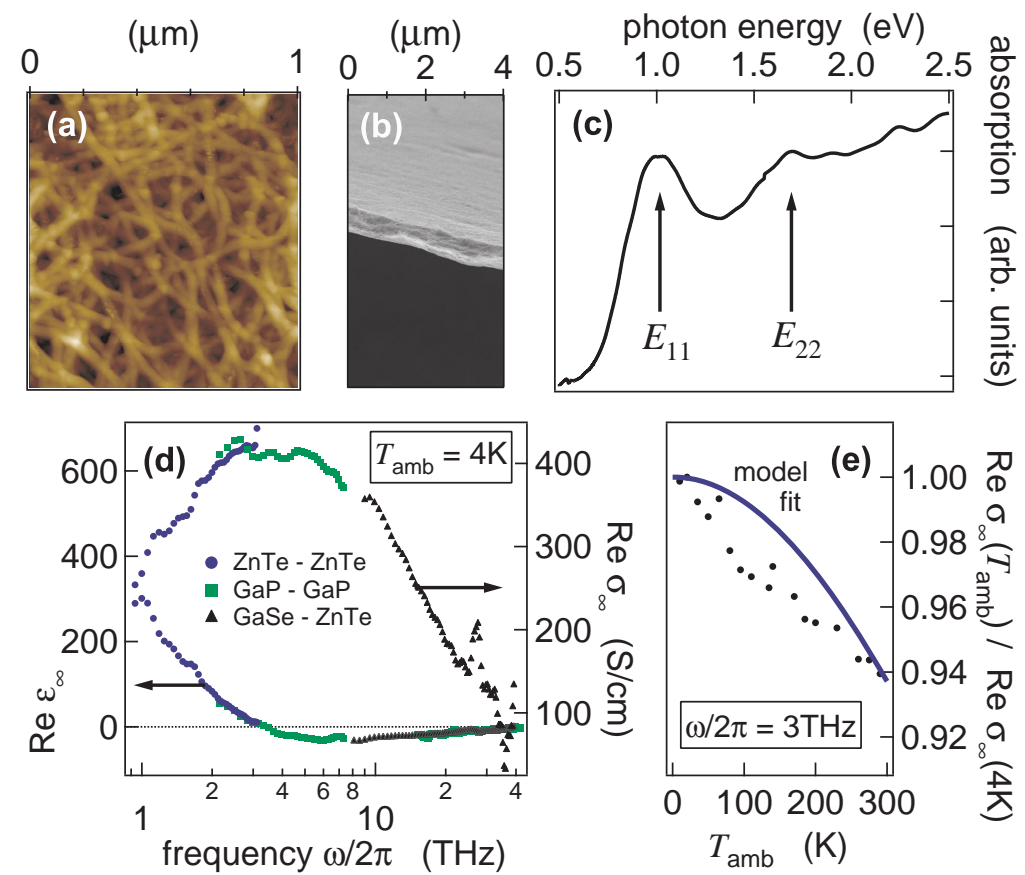

Figure 1. (a) Topography of our NT sample taken with an atomic force microscope resolving NT bundles. (b) Side view of the NT film obtained with a scanning electron microscope. (c) Optical absorption spectrum of the NT sample showing excitonic resonances $E_{11}$ at $1 \mathrm{eV}$ and $E_{22}$ at $1.6 \mathrm{eV}$. (d) Real parts of the measured dielectric function $\varepsilon_{\infty}$ and conductivity $\sigma_{\infty}$ of the unexcited sample with " $\infty$ " denoting an infinite pump-probe delay which is equivalent to the situation without pump pulse. Different marker colors in this panel represent the crystal pairs used for THz generation and detection. Note that both physical quantities $\varepsilon_{\tau}$ and $\sigma_{\tau}$ at pump-probe delay $\tau$ connect via $\varepsilon_{\tau}=1-c Z_{0} \sigma_{\tau} /(\mathrm{i} \omega)$ where $Z_{0} \approx 377 \Omega$ is the vacuum impedance. (e) $\operatorname{Re} \sigma_{\infty}$ as a function of ambient temperature $T_{\text {amb }}$ together with a fit according to Eq. (1) for fit parameters $\bar{\mu}=0$ and $\Delta \mu=120 \mathrm{meV}$.

\section{EXPERIMENTAL}

Our THz spectrometer based on Ti:sapphire lasers is either driven by laser pulses of 10-fs duration, 790-nm center wavelength, 10-nJ energy, and 75-MHz repetition rate from an oscillator (Femtolasers M1) or by 20-fs, 800-nm, $600-\mu \mathrm{J}, 1-\mathrm{kHz}$ pulses from an amplifier system (Femtolasers Femtopower Pro). The major part of the laser output is used to excite the NT sample whereas the remaining part is focused onto a ZnTe, GaP, or GaSe crystal to generate ultrashort and spectrally broad $\mathrm{THz}$ pulses by difference-frequency mixing. ${ }^{11}$ After transmission through the NT sample, the THz electric field is detected by electro-optic sampling in a $300-\mu \mathrm{m}$ thick ZnTe or $\mathrm{GaP}$ crystal. ${ }^{12,13}$ In order to cover the frequency window from 1 to $40 \mathrm{THz}$, we use various pairs of emitter and detection crystals, see frequency range covered in Fig. 1. For measurements at low temperatures, the sample is kept in a microscope cryostat (Cryovac Konti) equipped with diamond windows.

The NT sample is prepared similar to the procedure described in Ref. ${ }^{14}$ and details of our sample preparation and characterization are given in Ref. ${ }^{15}$. First, NTs grown by the high-pressure CO (HiPCO) method are dispersed in a 2-\% sodium-cholate solution. Centrifuging results in a supernatant that is used to produce a suspension containing a substantial amount of isolated NTs surrounded by sodium cholate molecules. After vacuum-filtering onto a filtration membrane and washing away the surfactant with purified water, the membrane is dissolved, and the remaining NT film is transferred to a diamond substrate. Subsequently, the film is annealed at $600^{\circ} \mathrm{C}$ under a constant flow of argon for one hour to remove most of the remaining contaminants. Finally, the film is capped by $\approx 1 \mu \mathrm{m}$ of parylene, in order to prevent NT oxidation triggered by laser radiation of the pump pulse. ${ }^{16}$

Figure 1(a) shows an image of the sample surface taken by an atomic force microscope resolving individual NT bundles. Note that these bundles are bent with a mean radius of curvature of $\sim 100 \mathrm{~nm}$. Figure 1(b) has 
been obtained by scanning electron microscopy and reveals that the NT film has a thickness of $\sim 700 \mathrm{~nm}$. The NTs of our sample have a diameter distribution that extends from about 0.8 to $1.2 \mathrm{~nm}$, and roughly $2 / 3$ of all NTs are large-gap tubes. ${ }^{17}$ In contrast to our previous estimation of the space filling factor based on the measured optical density and the absorption coefficient, ${ }^{15}$ we rely here on atomic-force micrographs, like the one in Fig. 1(a), which suggest a NT space-filling fraction $\mathcal{F}$ on the order of 0.2 (a value similar to Ref. ${ }^{15}$ ). Note that in any case the filling factor is not used directly in the subsequent calculations and hence does not affect our findings and interpretations presented further below. Figure 1(c) shows an absorption spectrum of the sample in the visible and near-infrared spectral range. Peaks labeled $E_{11}$ and $E_{22}$ arise from the first and second excitonic resonance in the large-gap tubes. ${ }^{18}$ Below $0.6 \mathrm{eV}$, the small-gap and metallic NTs can still absorb photons whereas the large-gap NTs are transparent. ${ }^{19}$

\section{RESULTS AND DISCUSSION}

In the following, our findings regarding the FIR absorption mechanism of the NT sample are presented where temperature dependent measurements of $\mathrm{THz}$ transmission under equilibrium conditions and pump-induced transmission changes are very well explained by a rather simple theoretical modelling. Subsequently, it is shown that the time-resolved response of the NTs underlines the photogeneration of strongly bound excitons to be the dominant process in large-gap tubes. Finally, polarization dependent experiments exhibit charge localization effects which are attributed to impurities and defects in the tube structure.

\subsection{Mechanism of far-infrared absorption}

In contrast to the visible spectral range, the microscopic mechanism of the far-infrared absorption is still unclear: The broad absorption feature at about $4 \mathrm{THz}$ was first assigned to optical interband transitions across the electronic energy gap of small-gap NTs as illustrated in Fig. 2(a). However, for an electronic transition with such low energy, this peak shows a surprisingly weak temperature dependence. ${ }^{20}$ As a consequence, phonons ${ }^{21}$ and, as sketched in Fig. 2(b), particle plasmons ${ }^{22}$ were suggested as alternative mechanisms.

Figure 1(d) shows the real parts of the conductivity and dielectric function $\operatorname{Re} \sigma_{\infty}(\omega)$ and $\operatorname{Re} \varepsilon_{\infty}(\omega)$, respectively, of the unexcited sample obtained from our time-domain measurements. $\operatorname{Re} \sigma_{\infty}(\omega)$ quantifies how strongly the sample absorbs light of frequency $\omega / 2 \pi$. It exhibits a broad peak centered at a resonance frequency $\omega_{\text {res }} / 2 \pi$ of roughly $4 \mathrm{THz}$ in agreement with previous reports. ${ }^{6-9,20-22,24}$ As seen in Fig. 1(e), this FIR absorption peak decreases only by less than $10 \%$ when the ambient temperature $T_{\mathrm{amb}}$ is raised from liquid helium to room temperature, 4 to $300 \mathrm{~K}$. Furthermore, an additional reduction of the conductivity peak occurs when the sample is excited, i.e. heated by an ultrashort pump pulse, which is demonstrated by the negative $\operatorname{Re} \Delta \sigma_{\tau}$ for several pump-probe delays $\tau$ given in Fig. 2(c). Furthermore, Figs. 2(c) and (d) show also that this pump-induced signal decays uniformly on a time scale of several picoseconds.

For a moment, we assume that the broad peak in Fig. 1(d) was due to a plasmon resonance. An elegant way to check the validity of this assumption is to excite the sample with a visible pump pulse. As explained in detail in Ref. ${ }^{23}$, the electrons inside a NT would feel an additional repulsive force exerted by the surface charges which are induced by the probing $\mathrm{THz}$ electric field, see Fig. 2(b). Consequently, a shift of this 4-THz peak and, thus, a derivative-like structure of the pump-induced changes Re $\Delta \sigma_{\tau}$ [Fig. 2(e)] are expected in case of a particle-plasmon resonance. Such a transient blue-shift was indeed observed, for instance, in GaAs nanowires in which the pump pulse increased the plasma frequency. ${ }^{25}$ However, the pump-probe data of our NTs as seen in Fig. 2(c) do not show any change in sign below $15 \mathrm{THz}$ and therefore clearly exclude a particle-plasmon based absorption mechanism.

In Ref. ${ }^{23}$, we developed a microscopic model which shows that the overall reduction of the FIR absorption peak of the pump-excited sample is a clear fingerprint of interband transitions blocked by transient hot electrons. Given that the model of independent electrons is valid in the small-gap tubes, the Fermi function $f_{\mu T}(\epsilon)=$ $\left\{\exp \left[(\epsilon-\mu) / k_{\mathrm{B}} T\right]+1\right\}^{-1}$ describes the occupation of a single-electron Bloch state with energy $\epsilon$. Here, the chemical potential $\mu$ is determined by the band structure, the electronic temperature $T$, and the number of electrons within an individual NT. Note that $\mu$ may vary from tube to tube since we have fixed the $\epsilon$ origin at the gap center for each tube with band gap energy $G$ [see Fig. 2(a)]. 

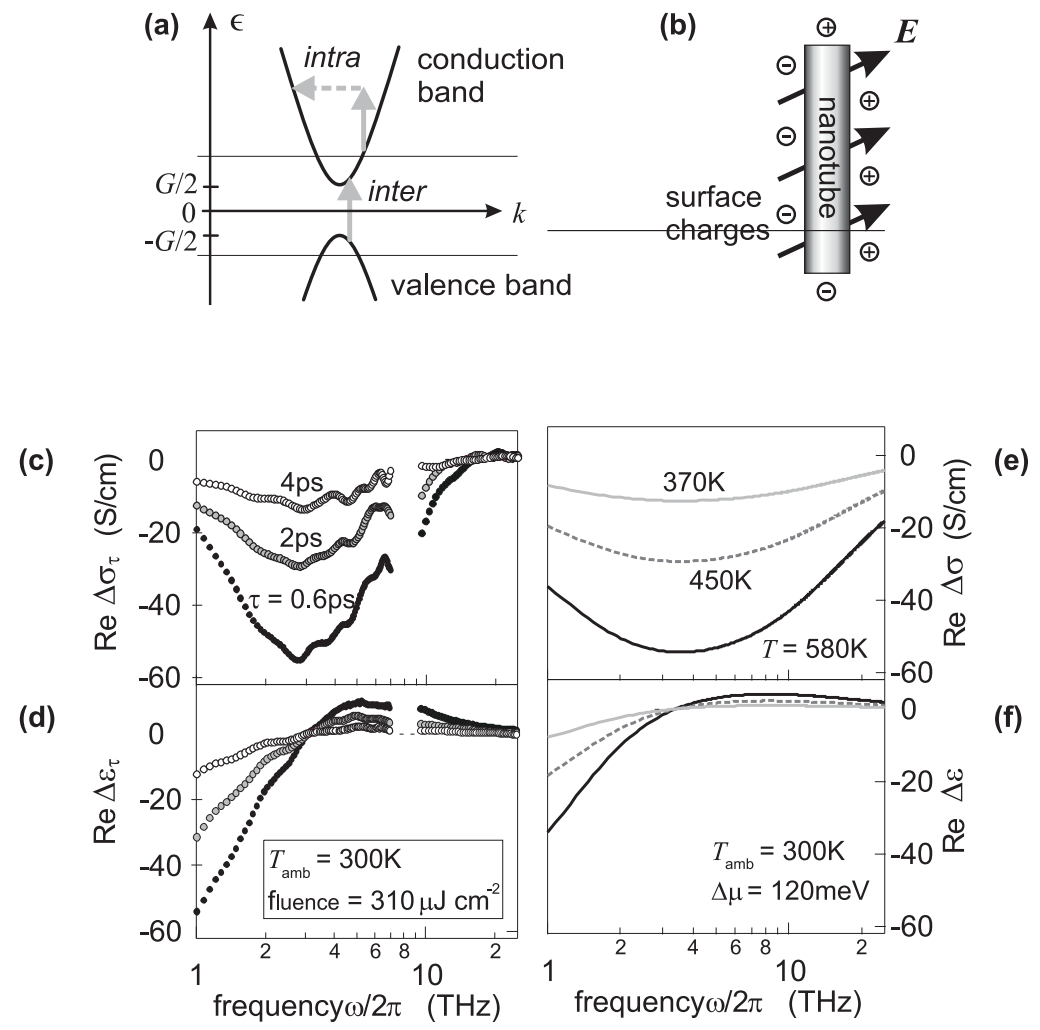

Figure 2. Schematic of proposed mechanisms explaining the far-infrared absorption of NTs. (a) Interband transitions across the band gap $G$ of a small-gap NT. An impurity-assisted intraband transition in the band structure $\epsilon(k)$ is also shown. (b) Particle-plasmon resonance. The probing electric field $\boldsymbol{E}$ induces charges on the NT surface which lead to an additional repulsive force on the electrons inside. (c,d) Pump-induced changes in the sample conductivity and dielectric function at several pump-probe delays $\tau$. (e,f) Calculated heat-induced changes in conductivity and dielectric function based on Eq. (1). ${ }^{23}$ Note the good qualitative agreement between experimental data and calculations.

The effective conductivity might be approximated by summing up the relevant contributions of all NTs of various types and orientations. ${ }^{23}$ Equivalently, one can also integrate over all values of $\mu, T$, and $G$ weighted with the combined probability distribution $p_{\mu T G}$. Assuming uncorrelated quantities, $p_{\mu T G}=p_{\mu} p_{T} p_{G}$, and one and the same electronic temperature $T$ in all tubes, the integration then reduces to

$$
\operatorname{Re} \sigma(\omega) \propto \frac{p_{G}}{3 \omega} \int \mathrm{d} \mu p_{\mu} F_{\mu T G}=: \frac{p_{G}}{3 \omega}\left\langle F_{\mu T G}\right\rangle
$$

where the Fermi function difference $F_{\mu T G}=f_{\mu T}(-G / 2)-f_{\mu T}(G / 2)$ with $G=\hbar \omega$ determines the strength of a transition between two levels separated by $G$, see Fig. 2(a). So-called interband transitions in such a two-level system mainly connect states near the top of the valence band and the bottom of the conduction band where the density of states is highest. This is corroborated by tight-binding calculations which predict the transition matrix elements to be dominant precisely for these transitions. ${ }^{26}$ The real part Re $\varepsilon$ of the dielectric function is obtained by a Hilbert transformation of $\operatorname{Re} \sigma$. In case of a sharply defined chemical potential, $\Delta \mu=0$, our model predicts a strong decrease of the conductivity with increasing temperature, in strong contrast to experimental data of Fig. 1(e). On the other hand, increasing $\Delta \mu$ gradually weakens the temperature dependence of $\operatorname{Re} \sigma .^{23}$ Finally, at $\Delta \mu=120 \mathrm{meV}$ assuming a Gaussian distribution $p_{\mu} \propto \exp \left[-(\mu-\underline{\mu})^{2} / 2 \Delta \mu^{2}\right]$, the modeled Re $\sigma$-versus-T curve matches the measured curve of Fig. 1(e) quite well. Possible origins of the $\mu$ variation are spurious doping and charge transfer between NTs with differing work function. ${ }^{27}$ 
(a)

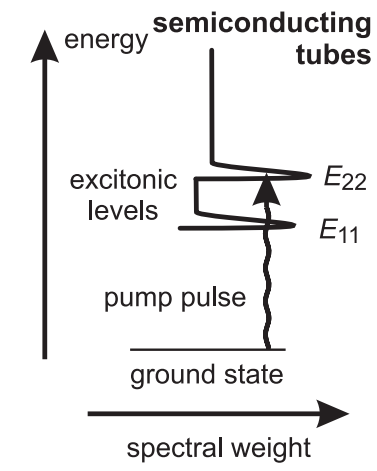

(c)

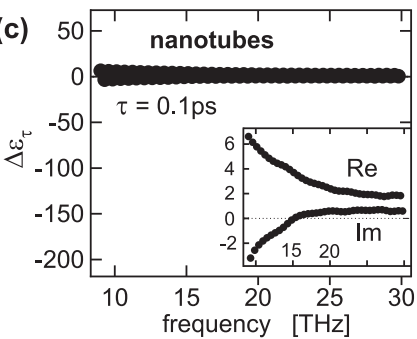

(b) metalic tubes
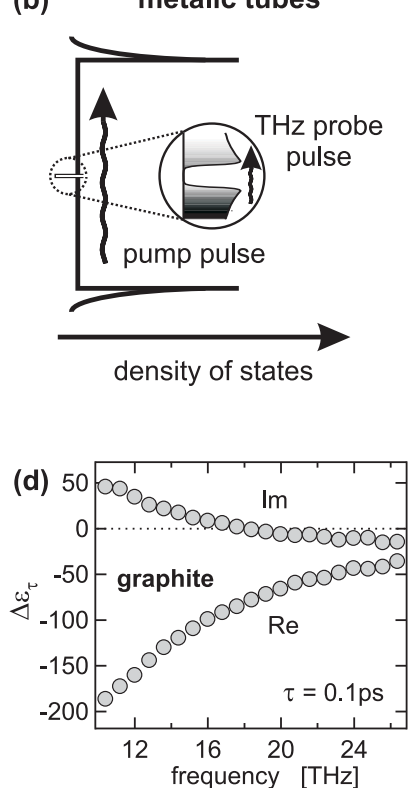

Figure 3. (a) Excitonic levels of a large-gap NT together with a visible pump pulse resonantly exciting the $E_{22}$ band. (b) Single-electron density of states of a small-gap NT with a magnification of the small band gap. Wiggled arrows mark transitions induced by the visible pump and the $\mathrm{THz}$ probe photons, respectively. The various gray scales represent different electronic occupation numbers. Pump-induced changes of the dielectric function of (c) carbon nanotubes and (d) graphite ${ }^{34}$ (in-plane dielectric function), both at $\tau=0.1 \mathrm{ps}$ after photoexcitation. Note the drastic difference of the absolute values and the sign as seen in the magnified graph in the inset.

\subsection{Photogeneration of excitons}

We next consider the changes of the dielectric function $\Delta \varepsilon_{\tau}$ of the NT sample after pump pulse excitation. Transient absorption measurements ${ }^{19}$ indicate that roughly half of the absorbed 1.6-eV pump photons resonantly populate the $E_{22}$-exciton bands of the large-gap tubes, which is illustrated in Fig. 3(a). The remaining portion is absorbed by small-gap and metallic NTs as sketched in Fig. 3(b). Figures 3(c) and (d) show the pump-induced changes $\Delta \varepsilon_{\tau}$ of our NTs and, for comparison, of an ultrathin sample (thickness $\leq 20 \mathrm{~nm}$ ) of the closely related graphite under comparable excitation conditions ${ }^{28}$ at a pump-probe delay of $\tau=0.1 \mathrm{ps}$. For the NTs, $\Delta \varepsilon_{\tau}$ is quite small and does not exhibit a distinct free-carrier response which should lead to $\operatorname{Re} \Delta \varepsilon_{\tau} \propto \omega^{-2}$. This is in striking contrast to the model-semiconductor $\mathrm{GaAs}^{29}$ and in particular also to graphite. As seen in Fig. 3(d) for the graphite sample, the two orders of magnitude larger and negative $\operatorname{Re} \Delta \varepsilon_{\tau}$ is indeed a clear signature of free charge carriers. On the other hand, the lack of such a response from the NT sample has important consequences. First, it strongly supports the assertion that excitons are the main products of photoexcitation of large-gap tubes. ${ }^{30,31}$ Since the internal excitation energies of these strongly bound electron-hole pairs ${ }^{32}$ exceed our probe energies by far, these excitons are invisible in our THz experiment. ${ }^{33}$ Second, the plasma frequency and the Drude scattering rate in the small-gap and metallic NTs do not change appreciably upon excitation. ${ }^{34}$ This can be traced back to their peculiar single-electron density of states [Fig. 3(b)] which is constant in metallic NTs or has a gap smaller than the thermal electronic energies occurring in our experiment. ${ }^{35,36}$ Moreover, parts of the electrons near the Fermi energy have been suggested to be localized due to disorder and thus do not contribute to a free-carrier response. ${ }^{37}$

\subsection{Localized and delocalized charge carriers}

For the further discussion of the origin of the pump-induced signal, we analyze the spectral structure of our data in Fig. 3(c), in particular the imaginary part of $\Delta \varepsilon_{\tau}$, which is plotted separately in Fig. 4(a). $\operatorname{Im} \Delta \varepsilon_{\tau}$ is positive 
(a)

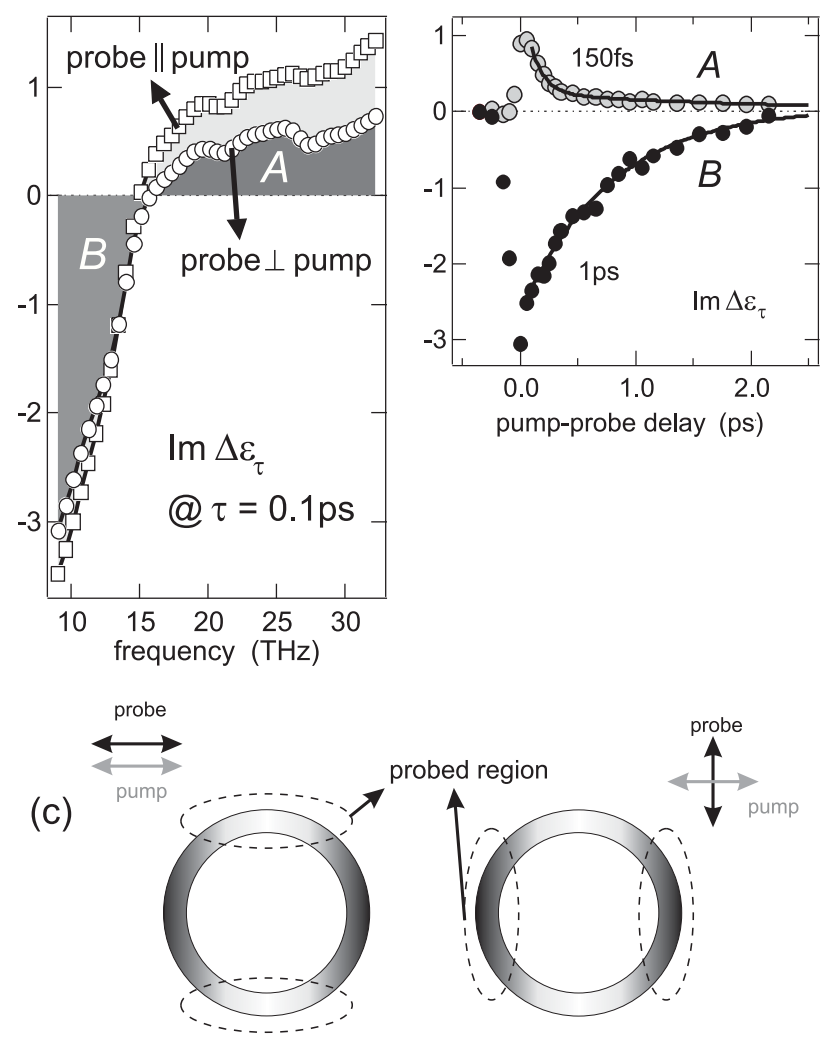

Figure 4. (a) Imaginary part of the pump-induced changes in the dielectric function $\operatorname{Im} \Delta \varepsilon_{\tau}$ at $\tau=0.1$ ps parallel and perpendicular to the pump polarization. The difference between both curves directly shows the pump-induced optical anisotropy. It is most pronounced in the imaginary part above $20 \mathrm{THz}$ where feature A dominates. Such dichroism is a direct consequence of excitations in the NTs which are localized on a length scale smaller than the radius of curvature of the bent tube. (b) Temporal decay of $\operatorname{Im} \Delta \varepsilon_{\tau}$ at $10.5 \mathrm{THz}$ (feature $B$ ) and $26 \mathrm{THz}$ (feature $A$ ). The decay of $B$ is well described by a single-exponential with a time constant of 1 ps whereas the decay of $A$ is fitted best to a double-exponential with time constants of $0.2 \mathrm{ps}$ and $1 \mathrm{ps}$, respectively. (c) Illustration of the origin of the pump-induced anisotropy, where the tube is represented by a closed ring with a certain radius of curvature. Since the polarizibility of NTs is much higher along the tube axis, the pump-induced signal is largest for parallel pump and probe polarizations because in this case the $\mathrm{THz}$ pulse mainly probes ring sections which have been excited by the pump pulse. On the contrary, for a perpendicular polarization configuration, basically unexcited ring sections are probed assuming the initial excitation is localized with respect to the ring radius. This results in a much weaker $\mathrm{THz}$ response. Consequently, the signal ratio of the two polarization configurations is a measure for the localization of the pump-induced excitations.

above $15 \mathrm{THz}$, which implies increased light absorption accordingly labeled " $A$ ". In contrast, $\operatorname{Im} \Delta \varepsilon_{\tau}$ is negative below $15 \mathrm{THz}$, which means increased light transmission (bleaching) and is labeled " $B$ ". Figures $4(\mathrm{a})$ and (b) show that $A$ and $B$ arise from distinct products in the photoexcited NTs. $A$ and $B$ exhibit significantly different temporal decays [see Fig. 4(b)]: At early times, $A$ decreases much faster than $B$. Furthermore, $A$ and $B$ respond differently to a change in the probe pulse polarization [see Fig. 4(a)]: The pump-induced dichroism with "II" and " $\perp$ " denoting configurations of parallel and perpendicular pump and probe polarizations, respectively, is defined as

$$
D=\frac{\operatorname{Im} \Delta \varepsilon_{\tau}^{\|}}{\operatorname{Im} \Delta \varepsilon_{\tau}^{\perp}}
$$

and found to be $\sim 1.1$ for feature $B$ but $\sim 1.5$ for feature $A$.

Such transient optical anisotropy has been previously observed for visible probe pulses ${ }^{31}$ and relies on the greatly reduced NT polarizability perpendicular to the tube axis. ${ }^{38,39}$ As a consequence, the probe pulse is most 
(a)

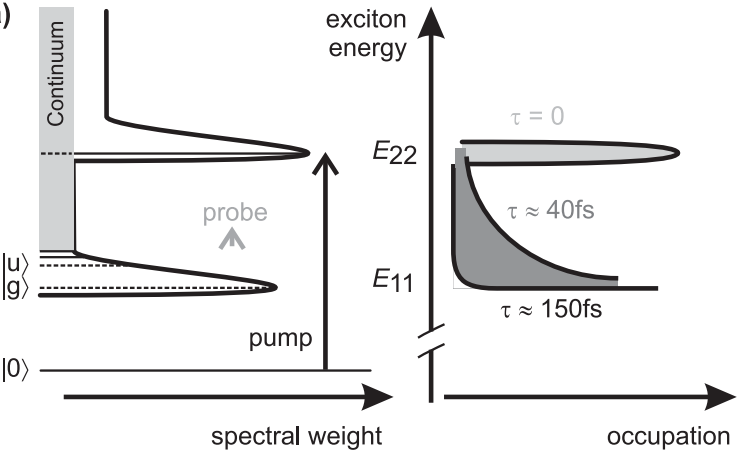

(b)

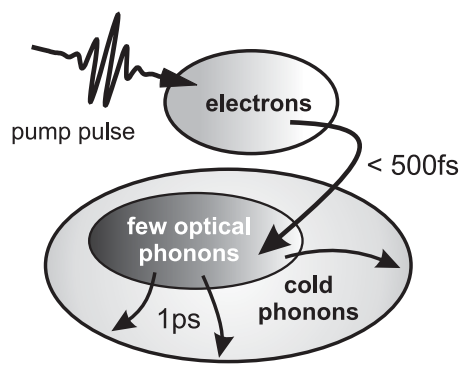

Figure 5. Illustrations of the pump-induced $\mathrm{THz}$ response of the NTs and the respective time scales. (a) Population relaxation of the excitonic levels. The pump pulse excites the $E_{22}$ exciton followed by a rapid redistribution of the exciton population with a 40 -fs time constant. ${ }^{30}$ Note that the excitonic energy has to be conserved in this process. Feature $A$ is assigned to transitions between higher-lying excitonic levels with small excitation energy. The excitonic excess energy and, therefore, feature $A$ decay with a 150 -fs time constant due to heat transfer from the excited excitons to the optical phonons until the exciton is in its ground state. The $\mathrm{THz}$ photon energy amounts to about half of the transition energy between the $\mathrm{g}$ and $\mathrm{u}$ levels. (b) The initially excited and hot electrons couple to only few, but strongly coupled optical phonon modes resulting in significant heating of these phonons. Only subsequently these hot phonons dissipate their energy to the remaining cold phonons, such a process is frequently called phonon bottleneck.

sensitive to tubes parallel to the probe polarization. Likewise, the pump pulse is absorbed most effectively by tubes parallel to the pump polarization. Therefore, parallel pump and probe polarizations should give a larger pump-induced signal than in the perpendicular case. This effect is largest if the pump-induced excitations have a spatial extent much smaller than the mean radius of curvature $R_{\mathrm{C}} \sim 100 \mathrm{~nm}$ of the bent NT leading to a dichroism with the maximal value of $3 .{ }^{40}$ On the other hand, a dichroism of 1 is attained when the excitations are spatially delocalized with respect to $R_{\mathrm{c}}$. Therefore, the pump-induced dichroism of $A$ and $B$ provides direct evidence of the existence of localized and delocalized electrons in the bent NTs. Anderson localization has been proposed previously by scanning tunneling spectroscopy. ${ }^{10} \mathrm{~A}$ simple estimate shows that the excitations giving rise to signal $B$ have an extent twice as large as those giving rise to $A{ }^{41}$ Note that the transient dichroism remains constant for pump-probe delays $\tau \leq 1 \mathrm{ps}$ showing that carrier diffusion is negligible during the first picosecond after sample excitation. ${ }^{42}$ The 0.2 -ps time constant for the fast initial decay of feature $A$ in Fig. $4(\mathrm{a})$ is remarkable since electronic thermalization takes place on the same time scale. ${ }^{43}$ We explain this finding by an increased absorption of localized charge carriers before they have thermalized. ${ }^{44}$

As far as the origin of the bleaching feature $B$ is concerned, phononic contributions are excluded, since no broad and optically active phonon bands are predicted in the probed spectral region. ${ }^{45}$ Consequently, $B$ must arise from electronic transitions. Stimulated emission from excited excitonic levels in large-gap tubes can be ruled out because the lowest $E_{11}$ state is attained within 40 fs after optical excitation ${ }^{30}$ (see Fig. 5(a)). We conclude that $B$ is due to metallic and small-gap tubes. The single-electron band-structure approach in Fig. 3(b) can qualitatively explain the observed effect: After pump pulse absorption, the excited electrons thermalize to a Fermi-Dirac distribution within $\approx 0.2$ ps. $^{43}$ This results in additional electrons and holes around the Fermi energy. These hot carriers block originally possible optical transitions connecting the van Hove singularities near the band gap. The contributions of various tube types lead to an inhomogeneously broadened bleaching feature $B$ as seen in our experiment. ${ }^{26}$

Finally, the 1-ps time constant measured for the $B$ and the final $A$ decays in Fig. $4(\mathrm{~b})$ reflects the lifetime of the excited electron distribution of NTs contributing to the signal. As illustrated in Fig. 5(b), the cooling of such hot electrons occurs via phonon emission leading to a non-equilibrium population of only few vibrational lattice modes able to scatter electrons. This effect is a general occurence in solids with a Fermi surface that allows only a restricted number of electronic wavevector changes. Examples are quasi-one dimensional conductors and graphite. ${ }^{34}$ Remarkably, the cooling of localized $(A)$ and delocalized $(B)$ carriers is equally fast indicating that the electron-phonon coupling is not significantly affected by the degree of carrier localization. 


\section{CONCLUSIONS}

$\mathrm{THz}$ spectroscopy has been used to study the far-infrared optics of single-wall carbon nanotubes. We have presented a simple model based on an ensemble of two-level systems which explains all observed qualitative features of the equilibrium and non-equilibrium FIR conductivity of small-gap NT ensembles. In particular, the controversially discussed weak temperature dependence of the FIR conductivity is consistent with the tubeto-tube variation of the chemical potential, which is about $0.1 \mathrm{eV}$ in our sample. Furthermore, our data lack

a distinct free-carrier response which is ascribed to the instantaneous generation of strongly bound excitons instead of free charge carriers as seen in graphite. Finally, the transient dichroism directly displays the existence of spatially delocalized and localized carriers. In summary, our time-domain and time-resolved THz spectroscopy experiments have contributed to a deeper understanding of the absorption mechanism and the charge carrier dynamics in carbon nanotubes.

\section{ACKNOWLEDGMENTS}

The carbon nanotube samples used have been produced by A. Hagen and in particular by C. M. Aguirre. Fruitful discussions with R. Martel, C. M. Aguirre, P. Desjadins, T. Hertel, and A. Hagen and financial support from the Deutsche Forschungsgemeinschaft through Sonderforschungsbereich 450 are gratefully acknowledged.

\section{REFERENCES}

[1] Dresselhaus, M. S., Dresselhaus, G., and Avouris, P., [Carbon Nanotubes: Synthesis, Structure, and Applications], Springer, Berlin (2001).

[2] Ouyang, M., Huang, J.-L., Cheung, C. L., and Lieber, C. M. Science 292, 702 (2001).

[3] Javey, A., Guo, J., Paulsson, M., Wang, Q., Mann, D., Lundstrom, M., and Dai, H. Phys. Rev. Lett. 92, 106804 (2004).

[4] Delaney, P., Choi, H. J., Ihm, J., Louie, S. G., and Cohen, M. L. Nature 391, 466 (1998).

[5] Snow, E. S., Perkins, F. K., Houser, E. J., Badescu, S. C., and Reinecke, T. L. Science 307, 1942 (2005).

[6] Kamaras, K., Itkis, M. E., Hu, H., Zhao, B., and Haddon, R. C. Science 301, 1501 (2003).

[7] Ruzicka, B., Degiorgi, L., Gaal, R., Thien-Nga, L., Bacsa, R., Salvetat, J.-P., and Forro, L. Phys. Rev. B 61, R2468 (2000).

[8] Borondics, F., Kamaras, K., Nikolou, M., Tanner, D. B., Chen, Z. H., and Rinzler, A. G. Phys. Rev. B 74, 045431 (2006).

[9] Itkis, M. E., Niyogi, S., Meng, M. E., Hamon, M. A., Hu, H., and Haddon, R. C. Nano Letters 2, 155 (2002).

[10] Gómez-Navorro, C., Pablo, P. J. D., Gómez-Herrero, J., Biel, B., Garcia-Vidal, F. J., Rubio, A., and Flores, F. Nature Mater. 4, 534 (2005).

[11] Huber, R., Brodschelm, A., Tauser, F., and Leitenstorfer, A. Appl. Phys. Lett. 76, 3191 (2000).

[12] Kampfrath, T., Nötzold, J., and Wolf, M. Appl. Phys. Lett. 90, 231113 (2007).

[13] Wu, Q. and Zhang, X. C. Appl. Phys. Lett. 70, 1784 (1997).

[14] Wu, Z., Chen, Z., Du, X., Logan, J. M., Sippel, J., Nikolou, M., Kamaras, K., Reynolds, J. R., Tanner, D. B., Hebard, A. F., and Rinzler, A. G. Science 305, 1273 (2004).

[15] Kampfrath, T., Perfetti, L., von Volkmann, K., Aguirre, C. M., Desjardins, P., Martel, R., Frischkorn, C., and Wolf, M. phys. stat. sol. (b) 244, 3950 (2007).

[16] Chan, S.-P., Chen, G., Gong, X. G., and Liu, Z.-F. Phys. Rev. Lett. 90, 086403 (2003).

[17] Hagen, A. and Hertel, T. Nano Lett. 3, 383 (2003).

[18] Wang, F., Dukovic, G., Brus, L. E., and Heinz, T. F. Science 308, 838 (2005).

[19] Ellingson, R. J., Engtrakul, C., Jones, M., Samec, M., Rumbles, G., Nozik, A. J., and Heben, M. J. Phys. Rev. B 71, 115444 (2005).

[20] Ugawa, A., Rinzler, A. G., and Tanner, D. B. Phys. Rev. B 60, R11305 (1999).

[21] Jeon, T.-I., Kim, K.-J., Kang, C., Maeng, I. H., Son, J.-H., An, K. H., Lee, J. Y., and Lee, Y. H. J. Appl. Phys. 95, 5736 (2004). 
[22] Akima, N., Iwasa, Y., Brown, S., Barbour, A. M., Cao, J., Musfeldt, J. L., Matsui, H., Toyota, N., Shiraishi, M., Shimoda, H., and Zhou, O. Adv. Mater. 18, 1166 (2006).

[23] Kampfrath, T., von Volkmann, K., Aguirre, C. M., Desjardins, P., Martel, R., Krenz, M., Frischkorn, C., Wolf, M., and Perfetti, L. Phys. Rev. Lett. 101, 267403 (2008).

[24] Kim, U. J., Liu, X. M., Furtado, C. A., Chen, G., Saito, R., Jiang, J., Dresselhaus, M. S., and Eklund, P. C. Phys. Rev. Lett. 95, 157402 (2005).

[25] Parkinson, P., Lloyd-Hughes, J., Gao, Q., Tan, H. H., Jagadish, C., Johnston, M. B., and Herz, L. M. Nano Lett. 7, 2162 (2007).

[26] Shyu, F. L. and Lin, M. F. J. Phys. Soc. Jpn. 71, 1820 (2002).

[27] Suzuki, S., Watanabe, Y., Homma, Y., Fukuba, S., Heun, S., and Locatelli, A. Appl. Phys. Lett. 85, 127 (2004).

[28] From absorption cross sections for $1.6-\mathrm{eV}$ photons ${ }^{46}$ we estimate that $\sim 10^{-4}$ photons per carbon atom are absorbed close to the surface of both the NT and the graphite sample.

[29] Huber, R., Tauser, F., Brodschelm, A., Bichler, M., Abstreiter, G., and Leitenstorfer, A. Nature 414, 286 (2001).

[30] Manzoni, C., Gambetta, A., Menna, E., Meneghetti, M., Lanzani, G., and Cerullo, G. Phys. Rev. Lett. 94, 207401 (2005).

[31] Sheng, C.-X., Vardeny, Z. V., Dalton, A. B., and Baughman, R. H. Phys. Rev. B 71, 125427 (2005).

[32] Wang, F., Dukovic, G., Brus, L. E., and Heinz, T. F. Science 308, 838 (2005).

[33] Hendry, E., Schins, J. M., Candeias, L. P., Siebbeles, L. D. A., , and Bonn, M. Phys. Rev. Lett. 92, 196601 (2004).

[34] Kampfrath, T., Perfetti, L., Schapper, F., Frischkorn, C., and Wolf, M. Phys. Rev. Lett. 95, 187403 (2005).

[35] Ehrenreich, H. and Cohen, M. H. Phys. Rev. 115, 786 (1959).

[36] von Baltz, R. and Escher, W. phys. stat. sol. (b) 51, 499 (1972).

[37] Hilt, O., Brom, H. B., and Ahlskog, M. 61, R5129 (2000).

[38] Spataru, C. D., Ismail-Beigi, S., Benedict, L., and Louie, S. G. Appl. Phys. A 78, 1129 (2004).

[39] Sfeir, M. Y., Wang, F., Huang, L., Chuang, C.-C., Hone, J., O’Brien, S. P., Heinz, T. F., and Brus, L. E. 306 (2004).

[40] We assume all NTs in our sample to form circles with radius $R_{\mathrm{C}}$ parallell to the sample surface. The pump pulse induces a polarizability change $\Delta \alpha(\varphi)$ of a short NT segment which is tilted by an angle $\varphi$ with respect to the probe beam polarization. As a consequence, the probe pulse induces a polarization change proportional to $E^{0} \Delta \alpha(\varphi) \cos \varphi$ which leads to a pump-induced signal $\Delta E_{\tau} \propto E^{0} \int_{-\pi / 2}^{\pi / 2} \mathrm{~d} \varphi \Delta \alpha(\varphi) \cos ^{2} \varphi$ along the probe beam polarization. For pump-induced excitations much smaller than $R_{\mathrm{c}}$, one has $\Delta \alpha(\varphi) \propto \cos ^{2} \varphi$ and $\propto \sin ^{2} \varphi$ for the $\|$ and $\perp$ configuration, respectively. This results in a pump-induced dichroism of 3. For delocalized excitations, $\Delta \alpha(\varphi)$ does not depend on $\varphi$ yielding a dichroism of 1 .

[41] Delocalization can be modeled by convoluting the perfectly localized $\Delta \alpha(\varphi)$ of Ref. ${ }^{40}$ with a Gaussian "smear-out" function of width $l_{\text {loc }} / R_{\mathrm{c}}$ where $l_{\text {loc }}$ is the localization length of the charge carriers.

[42] Perfetti, L., Kampfrath, T., Schapper, F., Hagen, A., Hertel, T., Aguirre, C. M., Desjardins, P., Martel, R., Frischkorn, C., and Wolf, M. Phys. Rev. Lett. 96, 027401 (2006).

[43] Hertel, T. and Moos, G. Chem. Phys. Lett. 320, 359 (2000).

[44] Mott, N. F. and Davis, E. A., [Electronic Processes in Non-Crystalline Materials], Clarendon, Oxford (1971).

[45] Ye, L. H., Liu, B.-G., Wang, D.-S., and Han, R. Phys. Rev. B 69, 235409 (2004).

[46] Islam, M. F., Milkie, D. E., Kane, C. L., Yodh, A. G., and Kikkawa, J. M. Phys. Rev. Lett. 93, 037404 (2004). 